\title{
3D Deformable Surfaces with Locally Self-Adjusting Parameters - A Robust Method to Determine Cell Nucleus Shapes
}

\author{
Margret Keuper* ${ }^{* \ddagger}$, Thorsten Schmidt ${ }^{* \ddagger}$, Jan Padeken ${ }^{\dagger \ddagger}$, Patrick Heun ${ }^{\dagger \ddagger}$, Klaus Palme Pl $^{\S \ddagger}$, \\ Hans Burkhardt ${ }^{* \ddagger}$ and Olaf Ronneberger* ${ }^{*}$ \\ * Chair of Pattern Recognition and Image Processing \\ Computer Science Department, Freiburg University, Germany \\ Email: keuper@informatik.uni-freiburg.de \\ ${ }^{\dagger}$ Max-Planck Institute of Immunobiology, Freiburg, Germany \\ ${ }^{\ddagger}$ Centre of Biological Signalling Studies (bioss), University of Freiburg, Germany \\ $\S$ Inst. of Biology II and Freiburg Inst. for Advanced Studies - FRIAS, University of Freiburg, Germany
}

\begin{abstract}
When using deformable models for the segmentation of biological data, the choice of the best weighting parameters for the internal and external forces is crucial. Especially when dealing with 3D fluorescence microscopic data and cells within dense tissue, object boundaries are sometimes not visible. In these cases, one weighting parameter set for the whole contour is not desirable. We are presenting a method for the dynamic adjustment of the weighting parameters, that is only depending on the underlying data and does not need any prior information. The method is especially apt to handle blurred, noisy, and deficient data, as it is often the case in biological microscopy.
\end{abstract}

Keywords-active contour; parameter estimation; cell segmentation

\section{INTRODUCTION}

For the analysis of inter- and intra cell signaling processes from 3D microscopic data, an exact knowledge about the anatomy of the individual cells is needed. Using active surfaces in order to perform segmentation or to fit models to the data is a widely employed method. In [2], an active surface model for the detection and segmentation of Drosophila Schneider cell (also called S2 cell) nuclei from strongly blurred 3D widefield microscopic data is presented. Because of the especially adapted force field, this method is able to deal with the low data quality caused by the microscopy technique as well as problem inherent difficulties like touching cells. Still, finding the best active surface parameters is crucial, and in most cases the same parameters do not work for different datasets. Thus, an automatic adjustment of these weighting parameters is desirable. For the special task of segmenting cell nuclei, it is also often the case that the contour of the object we want to segment is not fully visible because of bad contrast or staining artifacts. E.g cell nucleoli are often not stained and thus cause holes in the nucleus boundaries, whereas regions of dense chromatin result in very bright image regions and thus hamper a good segmentation. This is why we additionally want our method to locally adapt the weighting parameters for every surface point separately - thus promoting smooth surfaces where the data is lacking. The method we present here is based on the assumption, that the boundaries of the objects we want to segment are in some way similar over the whole object's surface. The external, data driven active surface forces are thus strongly weighted if this constraint is fulfilled. If a boundary estimate looks much different from the rest of the boundary, the data is considered deficient and high weights are assigned to the internal active surface forces. Thus we are replacing the classical low level weighting parameters by one high level parameter, which is the ratio of the boundary that is surely not missing in the recording.

The main difference to the active appearance models as e.g. presented in [5], where the relationship between model parameter displacements and residual errors is learned in a training step, is that for our method no prior knowledge about the concrete boundary appearance is included and no training has to be done. In [4], a dynamic combination of boundary and region information for 2D segmentation problems is presented, which is, as our approach, based on probability maximization. Unlike in our method, the dynamic weighting is there included into a level set framework and used to combine region and edge information in 2D color images. The internal forces are not dynamically regulated. In [3], a level set segmentation algorithm handling missing edges is presented, that is based on gradient flow. Unlike our algorithm, this method relies on a user-defined initial guess.

We are applying our method to the segmentation of two types of cell nuclei, to DAPI stained cultures of Drosophila S2 cells from 3D widefield microscopic recordings and to cells in a DAPI stained Arabidopsis Thaliana root tip recorded with a confocal laser scanning microscope (CLSM) (see fig. 1).

\section{Segmentation with 3D Active Surfaces}

Active surfaces are a common method for the segmentation of biological data. Given a rough estimate of the objects 

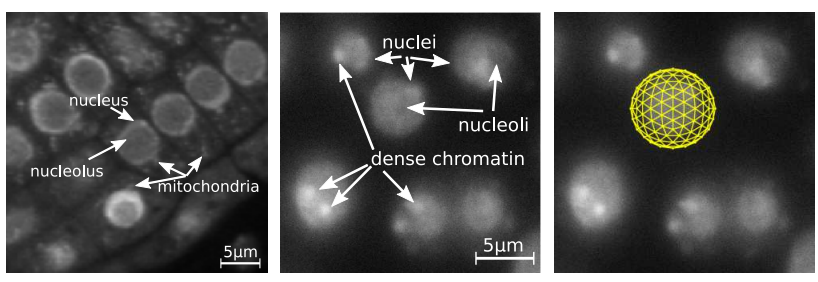

a) 3D CLSM recording of DAPI (b) DAPI stained Drosophila S2 (c) The same slice with the initia stained Arabidopsis Thaliana root tip cell nuclei from 3D wide field mi- active surface grid of one nucleus. cell nuclei in $x y$-view.

Figure 1. Raw data and initial surface grid.

position and size, i.e. centers $\mathbf{c}$ and radii $r$, an accurate fitting of the model to the underlying data can be performed.

The 3D active surface can be described as a function $\mathbf{X}$ which is placed on a 3D dataset $I$. Active surfaces have internal energies, depending only on the shape of the model itself, and are subjected to external energies coming from the underlying dataset to which the model shall be adapted. The total energy of an active surface is thus $E(\mathbf{X})=$ $E_{\text {int }}(\mathbf{X})+E_{\text {ext }}(\mathbf{X})$. The adaption takes place in minimizing this energy [1].

Mesh Design In 3D space, we need a mesh structure to sample the surface. As we focus on the segmentation of 2-sphere like objects, we choose a spherical grid for the segmentation. An equidistant sampling of the sphere can be done by an icosahedron structure. As we want to have a higher resolution, we are initializing our surfaces with a subdivided icosahedron, i.e. a triangle mesh, with 162 vertices $v_{i} \in \mathrm{V}$, where $\mathrm{V}$ is the set of all vertices with cardinality $|\mathrm{V}|$. Initially, all vertices $v_{i}$ are located at positions $\mathbf{x}_{i}$ that have distance $r$ from the object's center $\mathbf{c}$ (compare fig. 1(c)).

Internal Forces As $E_{\text {int }}$, the weighted first and second derivative of the surface are used, preventing the surface from stretching and bending too much. The minimization of $E$ leads to an Euler-Lagrange equation that can be considered as a force balance system [1]:

$$
\alpha \mathbf{F}_{\text {int }}+\beta \mathbf{F}_{\text {ext }}=0 .
$$

with the internal forces $\mathbf{F}_{\text {int }}:=\frac{\partial^{2} \mathbf{X}}{\partial \mathbf{s}^{2}}-\frac{\partial^{2}}{\partial \mathbf{s}^{2}} \frac{\partial^{2} \mathbf{X}}{\partial \mathbf{s}^{2}}=: \mathbf{F}_{\text {elasticity }}+$

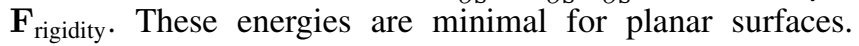
Therefore, on spherical structures, $\mathbf{F}_{\text {elasticity }}$ acts as shrinking force. To avoid shrinking effects, we allow $\mathbf{F}_{\text {elasticity }}$ only to pull the surface vertices on the tangent space of a sphere around the objects centers, i.e. we project the force onto this tangent space.

External Forces The external forces are responsible for the attraction of the active surface to the underlying data. For the segmentation of the nuclei, some application specific challenges are given. To allow for good segmentation results, we include the fact that our objects are sphere-like as a prior assumption into the force field. In [2], we have presented an external force field $\mathbf{F}_{\text {ext }}$ that is based on the

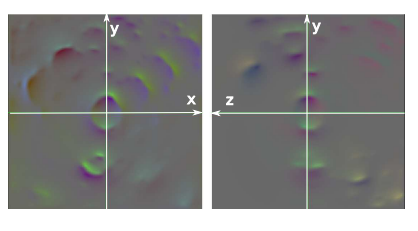

(a) $\mathbf{F}_{\text {ext }}$

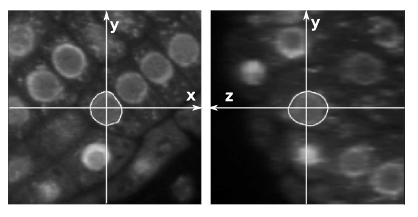

(c) $\alpha=0.5, \beta=0.5$
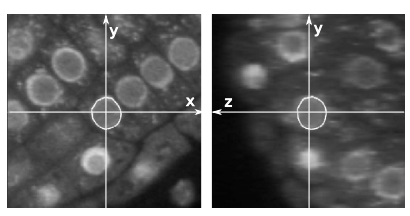

(b) $\alpha=0.9, \beta=0.1$

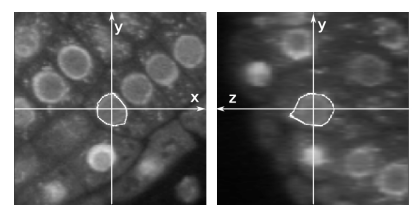

(d) $\alpha=0.1, \beta=0.9$
Figure 2. External force field and segmentation results with fixed parameters.

idea of projectiong the dataset gradients onto their radial component, and we have shown there, that this force field has some major advantages compared to standard gradient based force fields: it promotes 2 -spherical shapes, and its capture range is much larger. This is important, because of the touching cells in the datasets. Compare fig. 2(a) for an example.

Having these force fields defined, a good segmentation can still only be performed, if a suitable parameter set is given (compare fig. 2(b)-(d)).

\section{Dynamic Parameter Adjustment}

In most cases when segmentation of biological data is done, we are searching for certain boundary properties, e.g. edge profiles, texture, etc., which are in most cases homogeneous over the whole object surface. This is why we have based our method for the dynamic parameter adjustment on a homogeneity constraint on the boundary profile.

Since we are assuming 2-sphere like objects, we are considering the grayvalue profiles $\mathbf{r}_{\mathbf{x}_{i}} \in \mathbb{R}^{l}$ of the surface vertices $v_{i}$ in radial direction, where $l$ is the number of sampling points on the profile. For arbitrary shapes, the boundary profiles along the model surface normals should be taken instead. In every iteration step, we compare the possibly new profiles given external forces only to the profiles of the previous step. This is done to encourage the surface to move according to external forces whenever the resulting position fits into the average of the profiles. If a new vertex profile is similar to the other profiles, the underlying data contains valuable information and thus the external forces shall have high weights. If a new vertex profile is not similar to the other profiles, the underlying data is considered to be deficient. In these cases, we want to promote smooth, sphere-like model surfaces and thus assign high weights to the internal forces. This main idea is formalized as follows. To model the vertex similarities we have chosen a mul- 
tivariate normal distribution over the vertex profiles. The probability density function (PDF) is given by

$$
f\left(\mathbf{r}_{\mathbf{x}_{i}}\right)=\frac{1}{(2 \pi)^{l / 2}|\Sigma|^{1 / 2}} \cdot \mathrm{e}^{-\frac{1}{2}\left(\mathbf{r}_{\mathbf{x}_{i}}-\boldsymbol{\mu}_{\mathbf{r}}\right)^{T} \Sigma^{-1}\left(\mathbf{r}_{\mathbf{x}_{i}}-\boldsymbol{\mu}_{\mathbf{r}}\right)},
$$

where $\Sigma$ is the covariance matrix of all $|\mathrm{V}|$ profiles $\mathbf{r}_{\mathbf{x}_{i}}$ and $\mu_{\mathrm{r}}$ the expected value. Considering the joint distribution is necessary because of the high correlation of the grayvalues on the profiles. The PDF $f$ allows to compute the probability of a profile $P\left(\mathbf{r}_{\mathbf{x}_{i}}\right)$. The vertices lie on valid data with the probability $P(\mathrm{~B})$, which is the only, high level parameter we use. Since we assume that the majority of vertices, e.g. $80 \%$, lie on valid data, we compute a second PDF $g_{f}\left(\mathbf{r}_{\mathbf{x}_{i}}\right)$ to describe the appearance of the $80 \%$ of the profiles $\overline{\mathbf{r}}_{\mathbf{x}_{i}}$ that are most probable according to $f$, i.e. that describe valid boundary information:

$$
g_{f}\left(\mathbf{r}_{\mathbf{x}_{i}}\right)=\frac{1}{(2 \pi)^{l / 2}|\bar{\Sigma}|^{1 / 2}} \cdot \mathrm{e}^{\left.-\frac{1}{2}\left(\mathbf{r}_{\mathbf{x}_{i}}-\boldsymbol{\mu}_{\overline{\mathbf{r}}}\right)^{T} \bar{\Sigma}^{-1}\left(\mathbf{r}_{\mathbf{x}_{i}}-\boldsymbol{\mu}_{\overline{\mathbf{r}}}\right)\right)},
$$

where $\bar{\Sigma}$ is the covariance matrix of the $P(\mathrm{~B}) \cdot|\mathrm{V}|$ most probable profiles $\overline{\mathbf{r}}_{\mathbf{x}_{i}}$ according to $f$ and $\boldsymbol{\mu}_{\overline{\mathbf{r}}}$ is the respective expected value. With $g_{f}$, we can compute the conditional probability of a profile $\mathbf{r}_{\mathbf{x}_{i}}$ given the fact that it contains valid boundary information $p\left(\mathbf{r}_{\mathbf{x}_{i}} \mid \mathbf{B}\right)$, and thus with the Bayes Theorem the conditional probability of a profile $\mathbf{r}_{\mathbf{x}_{i}}$ to contain boundary information given its appearance:

$$
p\left(\mathbf{B} \mid \mathbf{r}_{\mathbf{x}_{i}}\right)=\frac{p\left(\mathbf{r}_{\mathbf{x}_{i}} \mid \mathbf{B}\right) \cdot P(\mathbf{B})}{P\left(\mathbf{r}_{\mathbf{x}_{i}}\right)} .
$$

For every vertex $v_{i}$, we compute its new position $\mathbf{x}_{i}^{\text {ext }}$ given external forces only and the according profile $\mathbf{r}_{\mathbf{x}_{i}^{\text {ext }}}$. Then, we compute $p\left(\mathbf{B} \mid \mathbf{r}_{\mathbf{x}_{i}^{\text {ext }}}\right)$.

The weights $\alpha_{i}$ and $\beta_{i}$ that are assigned to vertex $v_{i}$ in one iteration step are finally computed as:

$$
\alpha_{i}=1-p\left(\mathbf{B} \mid \mathbf{r}_{\mathbf{x}_{i}^{\text {ext }}}\right), \quad \beta_{i}=p\left(\mathbf{B} \mid \mathbf{r}_{\mathbf{x}_{i}^{\text {ext }}}\right),
$$

to ensure high weights for the external forces if $\mathbf{r}_{\mathbf{x}_{i}^{\text {ext }}}$ fits well to the majority of the profiles, and to assign low weights for the external forces if the profile does not. In most cases, we do not really want $\alpha_{i}$ to be 0 , ensuring no smoothness at all. Thus we are mostly adding a small $\epsilon$ to the $\alpha_{i}$.

During the evolution of the model, the determinant of the covariance matrix $|\Sigma|$ contains the information about the profile similarities. Once the model is attracted to the object boundaries, $|\Sigma|$ is small, whereas if the surface lies on heterogeneous regions, $|\Sigma|$ is large. This is why we are taking $|\Sigma|$ normalized by the maximum of its current value and its previous values as an additional convergence criterion, i.e. the evolution stops at step $t$, if the surface does not change or if $\frac{|\Sigma|_{t}}{\max \left(|\Sigma|_{1, \ldots, t}\right)} \leq 0.3$. The normalization has to be done because $|\Sigma|$ is maximal if the surface lies on heterogeneous data, e.g. is partially converged. If the surface is initialized in the background, one might start
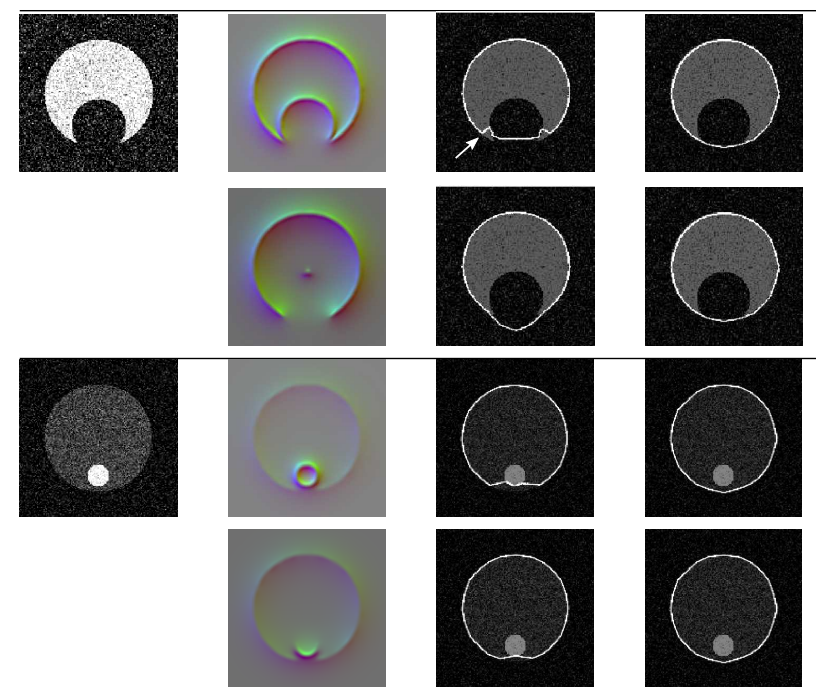

toy data

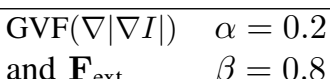

dynamic

weighting

Figure 3. Results for the toy examples with a classic GVF force field and $\mathbf{F}_{\text {ext }}$.

with a very low $|\Sigma|$. The value 0.3 is chosen heuristically. In our implementation, we compute the profile for each vertex over ten sampling points in radial direction from the object center. The resulting covariance matrices $\Sigma$ and $\bar{\Sigma}$ are thus $10 \times 10$ matrices.

\section{EXPERIMENTS}

The method was first tested on toy examples (see fig. 3) and then evaluated on two different types of cell nuclei: on cultures of Drosophila S2 cells that have been recorded with a widefield fluorescence microscope (393 cells) and on cells in a DAPI stained Arabidopsis Thaliana root tip recorded with a CLSM. The widefield recordings of the Drosophila cells have been processed without deconvolution, because the strong deconvolution artifacts in z-direction would have made a good segmentation impossible. The toy examples are designed to represent the problems that are present in the biological datasets. The first toy example is a sphere with a spherical hole, which should be ignored whenever encountered in our biological data, because it would probably correspond to a nucleolus. The second example is a sphere with a three times brighter sphere inside, corresponding to a spot of dense chromatin, which should not influence the segmentation neither. We have added gaussian noise to the two examples. With the dynamic weighting of the active surface forces, these toy examples have been well segmented, which, with fixed parameters, could not be done. For comparison, we have segmented the toy data with a classic force field $\operatorname{GVF}(\nabla|\nabla I|)$ and $\mathbf{F}_{\text {ext }}$.

On our real data, the method worked satisfactorily as well. The detection and radius estimation was done as in [2], and 
the surfaces were initialized from outside, with spheres with 1.5 times the estimated radius. Due to the strong blurring in z-direction it is hard to judge the segmentation of the Drosophila S2 cell nuclei in the lower regions, but the overall result seems reasonable for most of the 393 nuclei. See fig. 4 for some examples. On the left, you can see an overview of a dataset, on the right, the segmentation results for four example cells in orthogonal views.
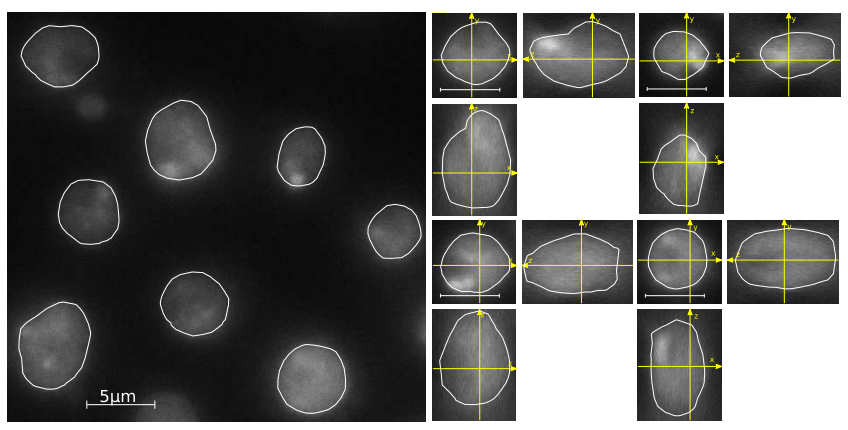

Figure 4. Segmentation results for the Drosophila S2 cell nuclei.

In fig. 5, the segmentation results of the proposed dynamic parameter estimation are displayed in direct comparison to the segmentation with fixed parameters. For the fixed parameters, we heuristically choose $\alpha=0.3$ and $\beta=0.8$ which actually shows good results as long as there are not too many bright chromatin spots in the data.
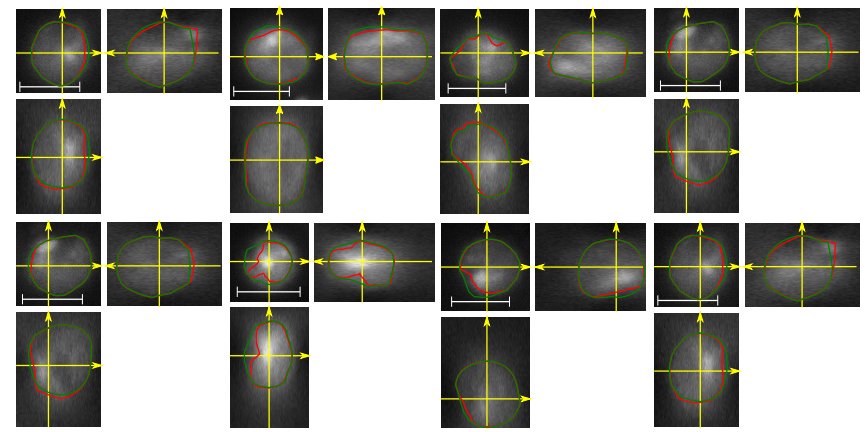

Figure 5. Segmentation results of eight cells in three orthogonal views. The white bar indicates as above the length of $5 \mu \mathrm{m}$. The contours found with fixed parameters are drawn in red, contours found with the proposed dynamic parameter estimation are drawn in green. The red contours are attracted to the bright chromatin spots if the spots are located near the boundary.

The segmentation of the Arabidopsis Thaliana nuclei is more difficult because of the dense tissue. The nuclei oftentimes touch one another and cell organelles touch the boundaries. Also, the more central the nuclei lie inside the root, the more difficult is the segmentation with our homogeneity based parameter weighting: as it can be seen in fig. 6, the nucleus boundaries become less and less homogeneous on the inner layers. Despite these facts, most of the nuclei could be properly segmented, see fig. 6 for an example slice, plotted in two orthogonal views. The colors correspond to the cell layer in the root.
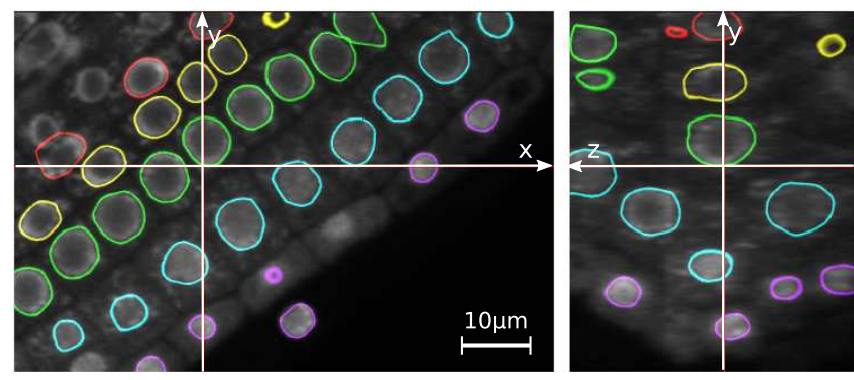

Figure 6. Segmentation results for Arabidopsis Thaliana root tip nuclei.

\section{CONCLUSION}

We have presented a probability based method to automatically adjust active surface parameters during the surface adaption process. The weighting parameters are regulated dynamically for each individual vertex based on its appearance - without the input of any prior knowledge. Thus, we avoid tedios parameter adjustment and allow for good segmentation results even in deficient data. For our noisy, biological datasets, the method has some major advantages compared to standard active surfaces with constant weighting parameters all over the surface: first, regions where the boundary information is missing can be closed smoothly, and second, gradients that originate from bright inner regions of the nuclei can be ignored, such that the correct boundary can be found.

\section{ACKNOWLEDGMENT}

This study was supported by the Excellence Initiative of the German Federal and State Governments (EXC 294).

\section{REFERENCES}

[1] C. Xu and J. L. Prince, Snakes, shapes, and gradient vector flow, IEEE Trans. Imag. Proc.,7/3, 1998, 321-345.

[2] M. Keuper, J. Padeken, P. Heun, H. Burkhardt and O. Ronneberger, A 3D Active Surface Model for the Accurate Segmentation of Drosophila Schneider Cell Nuclei and Nucleoli, Proc. of the ISVC, Springer LNCS, 2009, 865-874.

[3] A. Sarti, R. Malladi and J. A. Sethian, Subjective surfaces: A method for completing missing boundaries, PNAS, 97/12, 2000, 6258-6263.

[4] M. Allili and D. Ziou, An Approach for Dynamic Combination of Region and Boundary Information in Segmentation, Proc. of the ICPR, 2008, 1-4.

[5] T. F. Cootes,G. J. Edwards and C. J. Taylor, Active Appearance Models, IEEE Trans. on PAMI, 23/6, 2001, 681-685. 